\title{
Prognostic factors for mortality in patients with bullous pemphigoid: a meta-analysis
}

\author{
Yi-Di Liu ${ }^{1} \cdot$ Yan-Hong Wang ${ }^{2} \cdot$ Yi-Cong Ye ${ }^{3} \cdot$ Wen-Ling Zhao ${ }^{1} \cdot \mathrm{Li} \mathrm{Li}^{1}$
}

Received: 20 December 2016 / Revised: 3 March 2017 / Accepted: 8 March 2017 / Published online: 19 March 2017

(C) The Author(s) 2017. This article is published with open access at Springerlink.com

\begin{abstract}
Bullous pemphigoid (BP) is a chronic debilitating autoimmune blistering disease that frequently occurs in the elderly population. Previous studies have suggested a high morbidity and mortality associated with BP. However, relatively few studies have investigated prognostic factors of BP mortality, and they showed considerably various results. This meta-analysis aimed to quantitatively assess the association between several potential prognostic factors and risk of mortality in bullous pemphigoid. A comprehensive search was performed using Pubmed, Embase, and Cochrane Library. Cohort studies that assessed prognostic factors of BP mortality were included. Randomeffects model was utilized to calculate the pooled hazard ratio (HR). Publication bias was evaluated qualitatively by constructing a funnel plot and quantitatively by conducting Egger's test. 14 studies were included comprising 2499 patients. Combined HRs suggested that advanced age (HR $1.63,95 \%$ CI 1.34-1.97), presence of circulating antibodies (HR 1.77, 95\% CI 1.20-2.62), concomitant dementia (HR
\end{abstract}

Electronic supplementary material The online version of this article (doi:10.1007/s00403-017-1736-1) contains supplementary material, which is available to authorized users.

$\mathrm{Li} \mathrm{Li}$

lilipumch2007@sina.com

1 Department of Dermatology, Peking Union Medical College Hospital, Peking Union Medical College and Chinese Academy of Medical Science, Beijing 100730, China

2 Department of Epidemiology and Bio-statistics, Institute of Basic Medical Sciences, China Academy of Medical Sciences and Peking Union Medical College, Beijing 100730, China

3 Department of Cardiology, Peking Union Medical College Hospital, Peking Union Medical College and Chinese Academy of Medical Science, Beijing 100730, China
2.01, 95\% CI 1.22-3.33), and concomitant stroke (HR 1.86, 95\% CI 1.29-2.67) have an unfavorable impact on patient survival. Gender, disease extent, mucosal involvement, and indirect immunofluorescence result were not shown to be linked to mortality by our analysis. This study indicated that BP patients with older age, circulating antibodies, dementia, and stroke are at greater risk of mortality. Clinicians should be aware of this association and utilize this information in patient education and treatment process.

Keywords Bullous pemphigoid - Autoimmune blistering disease $\cdot$ Prognosis $\cdot$ Mortality $\cdot$ Survival

\section{Introduction}

Bullous pemphigoid (BP) is a chronic debilitating autoimmune blistering disease which has a high mortality and morbidity [28]. BP mainly affects the elderly people, causing symptoms, such as widespread tense blistering and itching. Previous studies reported an incidence of BP ranging from 2.5 to 42.8 cases per million per year $[2,11,14$, 23]. BP has been reported to be significantly associated with mortality, with morality rates ranging from 6 to $41 \%$ within the first year after diagnosis [1, 3-6, 12, 15, 22, 27, $29,30,32]$. BP patients also have increased mortality compared with their age and sex-matched controls in the general population $[2-4,12,14]$.

However, relatively few studies have investigated prognostic factors of BP mortality, with considerably various results. Old age has been shown to be related to poor outcome in BP in several studies [6, 12, 15, 27], but there are conflicting results on whether old age is an independent risk factor for mortality in BP [3, 21, 22]. In addition, poor general condition and low karnofsky scale [26] have 
often been associated with increased mortality [1, 15, 21, 29]. Interestingly, factor directly related to BP, such as the severity and extent of skin lesion, does not often predict survival [3, 12, 21, 22].

In addition, BP patients have complex comorbidity profiles, most notably neurological disorders as well as autoimmune, infectious and cardiovascular disorders [28]. Various studies have confirmed a strong association between BP and neurological diseases, particularly dementia, stroke, Parkinson disease, multiple sclerosis, and epilepsy [20]. BP is associated with development of autoantibodies directed against the hemidesmosomal BP autoantigens BPAG1 and BPAG2. BPAG1 has two isoforms: BPAG1-e is mainly expressed in skin, while BPAG1-a is expressed in central nervous system [20]. It is commonly suggested that an immunological cross-reaction between these isoforms could be the pathogenic reason. However, the prognostic value of co-existing neurological disorders as well as other comorbidities for BP mortality is unclear.

In addition, the sample sizes of these studies are often relatively small, leading to limited statistical power to detect true associations. In addition, there is still no systematic and quantitative assessment of published findings on this topic. We, therefore, conducted a meta-analysis to quantitatively assess the association between several potential prognostic factors and risk of mortality in bullous pemphigoid.

\section{Materials and methods}

This meta-analysis was conducted according to the Preferred Reporting Items for Systematic Reviews and MetaAnalyses (PRISMA) Statement protocol [25].

\section{Search strategy and study selection}

We performed an exhaustive search on Pubmed (from 1978 to November 2016), Embase (from 1974 to November 2016) and Cochrane Library (from 1994 to November 2016) to identify articles that studied the prognostic factors for BP mortality. There was no limit on human race, geographic region, language, or publication type. The major terms used included: "bullous pemphigoid", "mortality", "fatality", "survival", "death", "prognosis", and "prognostic". The following selection criteria were performed on Pubmed as an example:

1. bullous pemphigoid [Title] or bullous pemphigoid [Mesh Terms];

2. mortality or fatality or survival or death or prognosis or prognostic [All Fields];

3. 1 and 2 .
Studies eligible for inclusion in this meta-analysis met the following criteria: (a) cohort study design; (b) measure potential prognostic factors for BP mortality; (c) provide sufficient data for calculating the effect size with $95 \%$ confidence intervals (CIs); and (d) when the same author's data obtained from the same or overlapping patients in more than one publication, only the most recent report or the most complete one was selected in the analysis. We excluded case reports, reviews, meta-analyses, and letters. Studies investigating remission or relapse but not mortality of BP were excluded. Childhood bullous pemphigoid was not considered in the present study. Additional studies were identified by a manual search of the references of original studies. The literature search and inclusion of eligible articles was performed independently by two authors (Liu YD and Zhao WL) and disagreements were resolved at each step by consensus.

\section{Data extraction}

All data were extracted independently and crosschecked by two authors (Liu YD and Zhao WL). Data retrieved from the studies included authors, year of publication, patient source, number of patients included in the study, study type, follow-up time, and statistical results regarding the influence of prognostic factors on the risk of mortality. If both 1 year mortality and overall mortality were reported in a study, 1 year mortality was extracted for analysis.

\section{Quality assessment}

Quality assessment of each study was performed independently by the two authors (Liu YD and Zhao WL), using the Newcastle-Ottawa Scale (NOS) (http://www.ohri.ca/ programs/clinical_epidemiology/oxford.asp), which graded studies according to the quality of selection, comparability, and outcome of study participants. Studies that achieved seven or more stars on NOS were considered to be of high quality, four to six stars were medium quality, and fewer than four stars were poor quality [24]. Discrepancies were addressed by re-evaluation of the original article and discussion with a third author (Wang YH).

\section{Statistical methods}

For the quantitative aggregation of the survival results, we reported summary measures of effect size using a pooled hazard ratio (HR) with $95 \%$ confidence interval. If available, we extracted the risk estimates that underwent multivariate adjustment. For studies in which the HR corresponding to the $95 \%$ CI was not given directly, published data from original papers were used to calculate the HR according to the methods described previously [31]. In a 
few situations where there was not sufficient information for HR estimation, we calculated the relative risk (RR) with 95\% CI as a crude substitution of HR. Study heterogeneity was assessed using the Cochran $\mathrm{Q}$ test with $P$ value for significance set at 0.10 and the $I^{2}$ statistic with values of 25 , 50 and $75 \%$ considered as low, moderate, and high heterogeneity, respectively [13]. To estimate the pooled HR, random-effects model of DerSimonian and Laird was used [7]. Publication bias was evaluated with funnel plot and Egger's test $(P<0.05$ was considered statistically significant) [9]. The statistical analysis was performed by the STATA 14.0 software (StataCorp, College Station, TX, USA).

\section{Results}

\section{Study characteristics}

The preliminary literature search returned 660 studies matching the initial search criteria, as shown in Fig. 1. After screening, we identified 14 studies on ten Caucasian and four Asian population samples, including a total of 2499 patients after extracting overlapping patients between studies. The major characteristics of the 14 eligible publications were reported in Table 1 . Of the 14 studies, nine were retrospective cohort studies, and five were prospective cohort studies. Based on NOS, 13 studies were considered to be of high quality and one was of medium quality, as shown in Table 2. Most studies were conducted in major centers with an exception of one Swiss study which was population-based [6]. When there was identical or overlapping patient population between the 14 studies, we only included the study with the more complete population in the investigation of a specific prognostic factor of interest $[1,15,16,29]$.

\section{Quantitative synthesis}

Age

To investigate the effect of age on survival, we included the ten studies which compared survival outcome on elderly BP patients with younger age patient populations. In most studies, patients' median or mean age at the time of diagnosis was used as the cut-off value to categorize young and elderly patients, and the cut-off value ranged from 67 to 83 years across studies. The pooled HR of old age for mortality risk was 1.63 (95\% CI 1.34-1.97, $P<0.001)$. Significant heterogeneity was seen among studies $\left(I^{2}=86.6 \%\right.$, $P<0.001)$. In subgroup analysis by age cut-off value, studies using cut-off age $\geq 80$ (HR $1.42 ; 95 \%$ CI 1.16-1.73) and studies using cut-off age $<80$ (HR 2.14; 95\% CI 1.32-3.44) both showed statistical significance. Study heterogeneity remained moderate to high for studies using cut-off age $\geq 80$ $\left(I^{2}=90.4 \%, P<0.001\right)$ and studies using cut-off age $<80$ $\left(I^{2}=55.2 \%, P=0.063\right)$. Visual inspection of the funnel plot suggested obvious asymmetry, and this was further supported by Egger's test showing evidence for reporting bias $(P=0.001)$. "Trim and fill" method showed that five studies had to be added to correct funnel plot asymmetry, and
Fig. 1 Flowchart of studies included in the meta-analysis

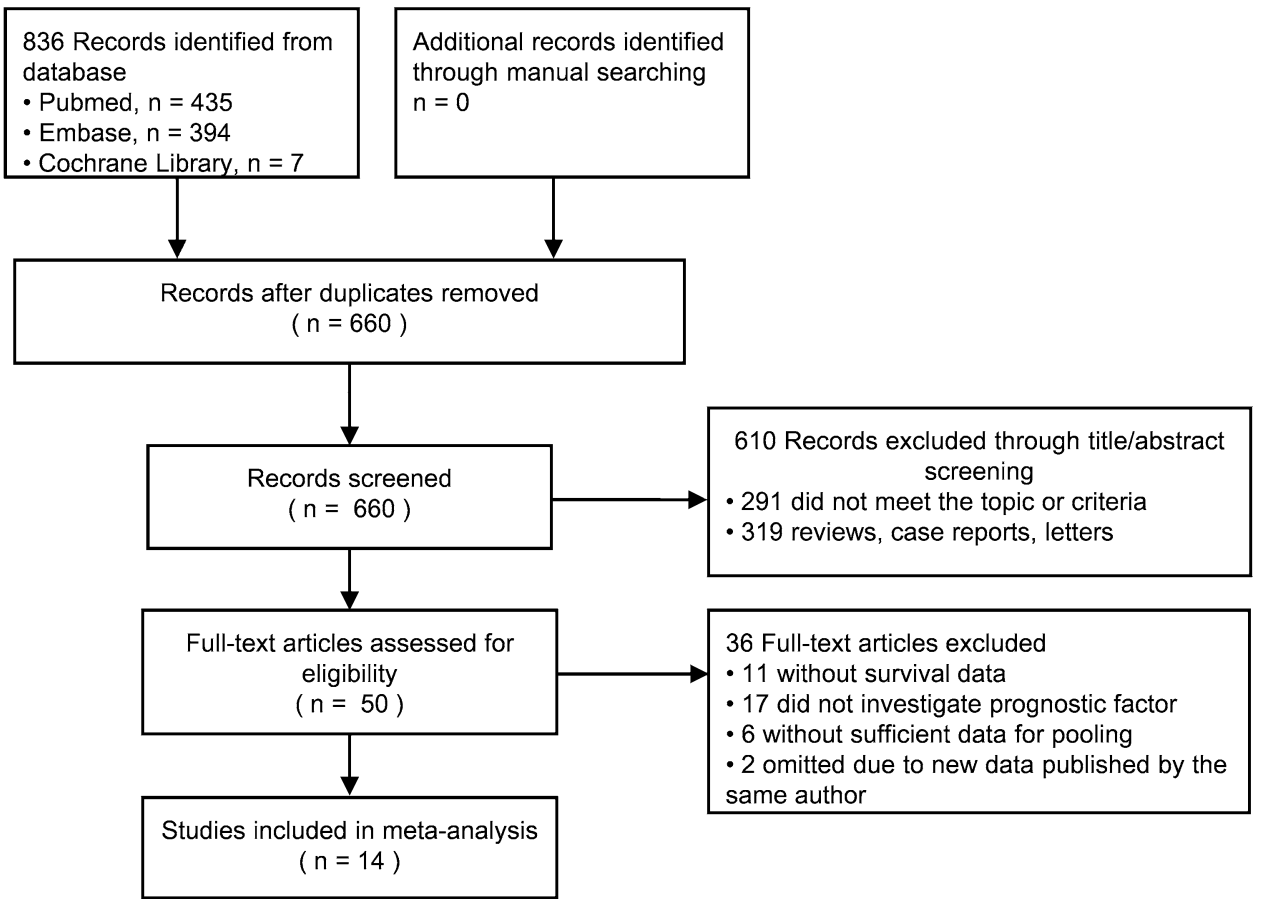


Table 1 Main characteristics of the eligible studies

\begin{tabular}{|c|c|c|c|c|c|c|c|c|}
\hline First author & Year & Country & Sample size & Study type & Type of care setting & HR estimation & Follow up time & Study quality \\
\hline Lee & 2014 & Korea & 168 & Retrospective & Tertiary center & 1-year mortality & 2.19 years $^{\mathrm{a}}$ & 8 \\
\hline Cai & 2014 & Singapore & 359 & Retrospective & Tertiary center & Overall mortality & 3 years & 8 \\
\hline Gual & 2014 & Spain & 101 & Retrospective & Tertiary center & 1-year mortality & 1 year & 9 \\
\hline $\mathrm{Li}$ & 2013 & China & 140 & Retrospective & Inpatient & Overall mortality & 3 years $^{\mathrm{a}}$ & 8 \\
\hline Zhang & 2013 & China & 94 & Retrospective & Inpatient & 1-year mortality & 1 year & 9 \\
\hline Cortés & 2012 & Switzerland & 60 & Retrospective & Inpatient & Overall mortality & 1 to 5 years & 8 \\
\hline Cortés & 2011 & Switzerland & 115 & Prospective & Outpatient & Overall mortality & 3 years & 9 \\
\hline Joly & 2009 & France & 312 & Prospective & Tertiary center & 1-year mortality & 1 year & 9 \\
\hline Parker & 2008 & USA & 223 & Retrospective & Outpatient and Inpatient & Overall mortality & 2.69 years $^{\mathrm{a}}$ & 6 \\
\hline Joly & 2005 & France & 170 & Prospective & Tertiary center & 1-year mortality & 1 year & 9 \\
\hline Rzany & 2002 & Germany & 369 & Retrospective & Inpatient & 1-year mortality & 1.9 years $^{\mathrm{a}}$ & 9 \\
\hline Joly & 2002 & France & 341 & Prospective & Tertiary center & 1 -year mortality & 1 year & 9 \\
\hline Roujeau & 1998 & France & 217 & Retrospective & Tertiary center & 6 month mortality & 1.5 year $^{\mathrm{a}}$ & 8 \\
\hline Bernard & 1997 & France & 94 & Prospective & Tertiary center & 1-year mortality & 1 year & 7 \\
\hline
\end{tabular}

${ }^{a}$ Follow up time in median follow up time

the pooled outcome was no longer statistically significant (HR 1.17; 95\% CI 0.96-1.42, $P=0.130$ ). Figures 2, 3, 4, 5, $6,7,8,9$ show the forest plots with summary measures of the associations between BP mortality and potential prognostic factors. Figure 10 illustrates the funnel plots of component studies in this meta-analysis. Table 3 summarizes the pooled results of potential risk predictors.

\section{Gender}

No significant impact was observed for BP mortality, and the pooled HR of male vs female was 1.01 (95\% CI $0.81-1.25, P=0.944$ ), as shown in Fig. 3. Low-to-moderate heterogeneity was observed between the studies $\left(I^{2}=41.6 \%, P=0.072\right)$. Visual inspection of the funnel plot did not reveal obvious asymmetry (Fig. 10), and it was confirmed by Egger's test $(P=0.706)$.

\section{Extensive disease}

The definition of disease extent varied among studies. Joly et al. have defined extensive disease as the occurrence of more than ten new blisters per day $[16,17]$. Two studies graded BP disease severity based on the percentage of body surface area involvement as follows: mild, $<10 \%$; moderate, $10-30 \%$; and severe, $>30 \%$ [21, 27]. Four studies defined localized disease as isolated lesions in one anatomical region, and generalized disease for patients with moderate-to-diffuse lesions in two or more regions $[3,12$, $22,29]$. For simplicity, we combined these eight studies and the pooled HR of extensive disease was 1.19 (95\% CI $0.97-1.46, P=0.102)$, as shown in Fig. 4. No heterogeneity was observed between the studies $\left(I^{2}=16.2 \%, P=0.302\right)$.
Visual inspection of the funnel plot could not rule out publication bias (Fig. 10), but the result of Egger's test was not significant $(P=0.233)$.

\section{Mucosal lesion}

The pooled HR of mucosal lesion vs no mucosal lesion was 1.00 (95\% CI 0.65-1.56, $P=0.992$ ), as illustrated in Fig. 5. The heterogeneity between the studies was low $\left(I^{2}=34.5 \%\right.$, $P=0.205)$. The funnel plot showed a lack of small size studies with positive association, suggesting probable publication bias (Fig. 10). Egger's test, however, failed to provide any evidence for small study effect $(P=0.079)$.

\section{Circulating BP autoantibody}

Because only six studies investigated the relationship between the presence of circulating BP antibodies and patient survival, we pooled the results of all these studies together, regardless of the detection method of antibodies. When a study performed both indirect immunofluorescence (IIF) and enzyme-linked immunosorbent assay (ELISA) or immunoblot, we utilized survival data based on ELISA (preferentially anti-BP180) or immunoblot instead of IIF, since ELISA result was reported to indicate disease activity [10]. The presence of circulating antibodies conferred a 1.77 fold increased risk (95\% CI 1.20-2.62, $P=0.004$ ), as shown in Fig. 6. The studies were considered homogenous $\left(I^{2}=0.0 \%, P=0.632\right)$. The funnel plot was roughly symmetrical (Fig. 10). Egger's test also failed to provide any evidence for small study effect $(P=0.859)$. In addition, we performed analysis for survival results based on positive IIF (HR $=1.17$; 95\% CI $0.75-1.81, P=0.488)$, as shown in 


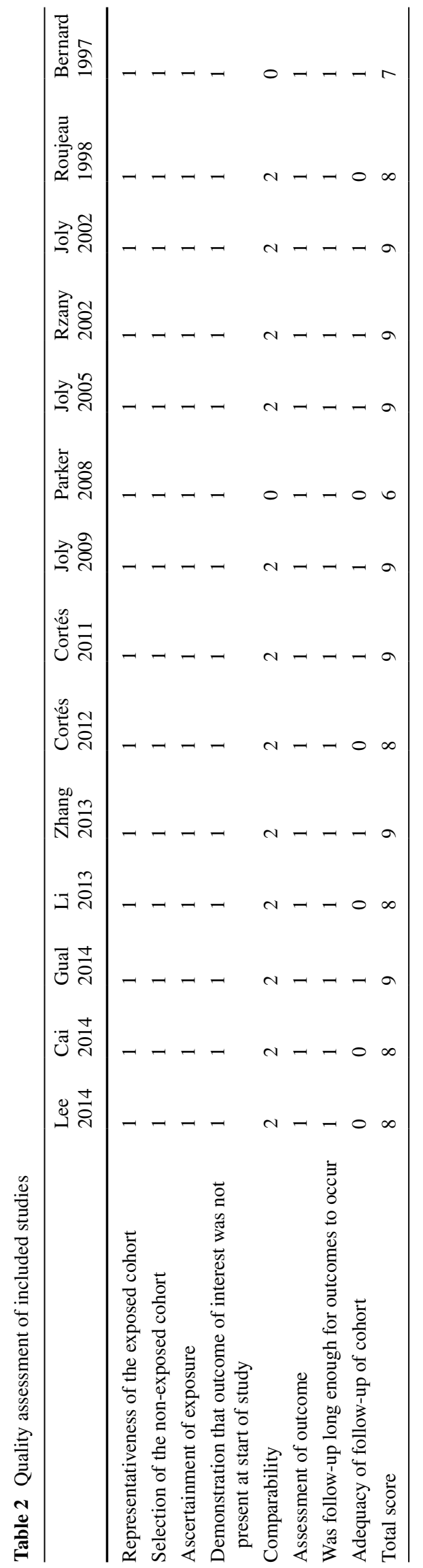

Fig. 7. The funnel plot was roughly symmetrical (Fig. 10), and Egger's test was not significant $(P=0.622)$.

\section{Dementia}

Patients with dementia (including Alzheimer's dementia and all other types of dementia) were twice more likely to die than patients who did not have dementia. The pooled HR was 2.01 (95\% CI 1.22-3.33, $P=0.006$ ), as shown in Fig. 8. There was moderate-to-high heterogeneity between the studies $\left(I^{2}=64.6 \%, P=0.015\right)$. The funnel plot was not perfectly symmetrical (Fig. 10), suggesting possible publication bias. Egger's test, however, failed to provide any evidence for small study effect $(P=0.853)$.

Stroke

Patients with stroke had nearly two-fold increased risk of death compared with patients who did not have this comorbidity. The pooled HR was 1.86 (95\% CI 1.29-2.67, $P<0.001$ ), as illustrated in Fig. 9. There was low-tomoderate heterogeneity between the studies $\left(I^{2}=40.8 \%\right.$, $P=0.133)$. Visual inspection of the funnel plot suggested probable reporting bias (Fig. 10), although Egger's test did not provide any evidence for small study effect $(P=0.277)$.

\section{Discussion}

In this meta-analysis of 14 studies that included 2499 subjects, we found that older age, presence of circulating antibodies regardless of detection method, co-existing dementia, and stroke were associated with an increased risk of BP mortality. No significant association was observed for gender, disease extent, mucosal involvement, or IIF result and survival.

In consistence with most studies included in the current analysis, we found no survival difference based on sex. However, age at diagnosis was associated with increased risk of death, with advanced age being a poor prognostic factor. Stratification of studies based on age cut-off value demonstrated that the effect size was more significant when the cutoff was lower, suggesting that the truly younger patients may have a better prognosis. Discrepancies in the age cut-off values, patient baseline characteristics, and treatment approaches between studies might contribute to the high level of heterogeneity. The pooled result did not remain comparable after "trim and fill" adjustment, suggesting that publication bias might have led to an overestimation of the effect of age on survival. However, the asymmetry in funnel plot may also simply be the play of chance, since only ten studies were included, or true heterogeneity existed because cut-off age varied greatly. Of note, the 


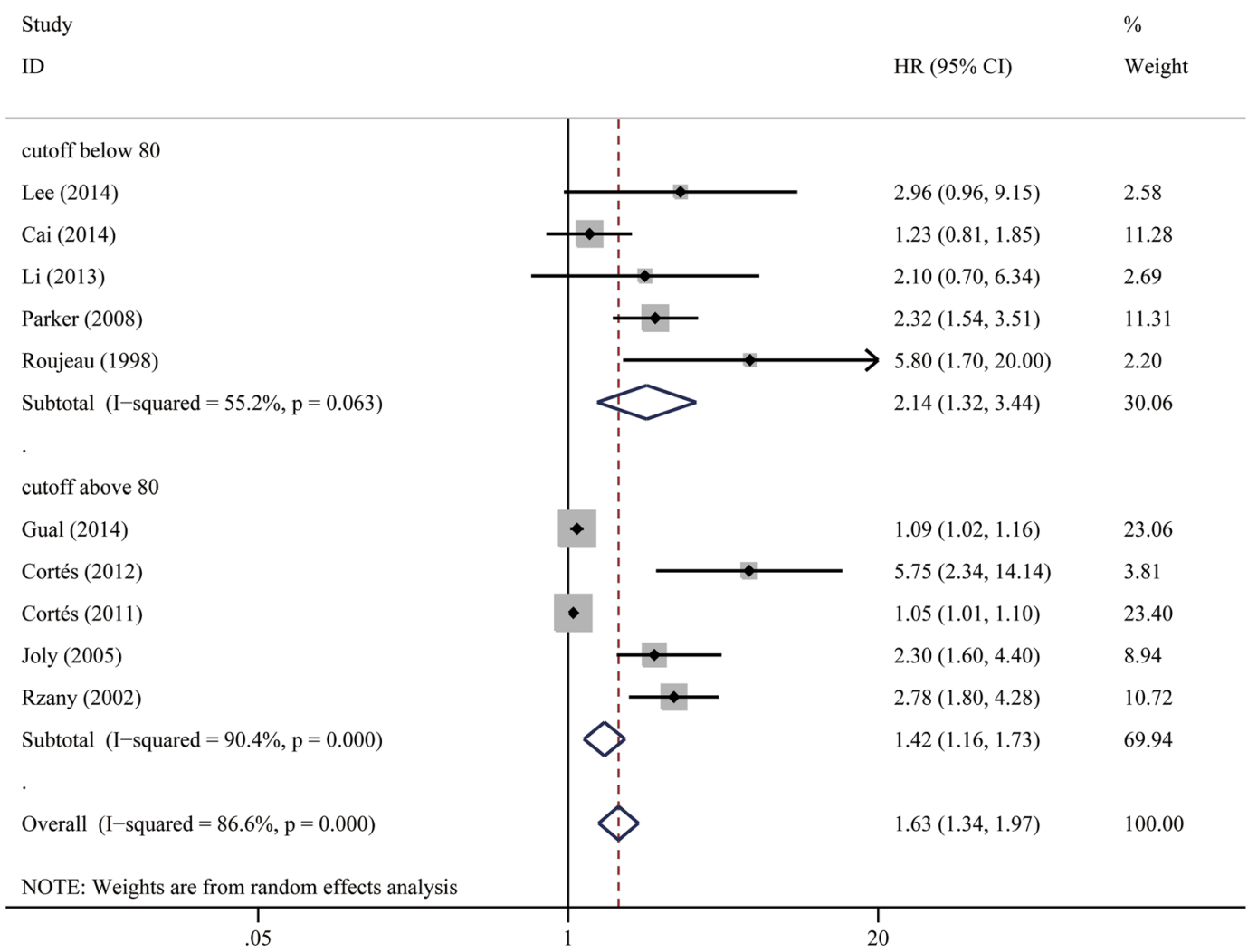

Fig. 2 Forest plots: association between bullous pemphigoid mortality and old age

Study
ID
Lee (2014)
Cai (2014)
Lual (2014)
Chang (2013)
Cortés (2011)
Parker (2008)
Joly (2005)
Rzany (2002)
Roujeau (1998)
Overall (I-squared = 41.6\%, $=0.072$ )

Fig. 3 Forest plots: association between bullous pemphigoid mortality and gender 


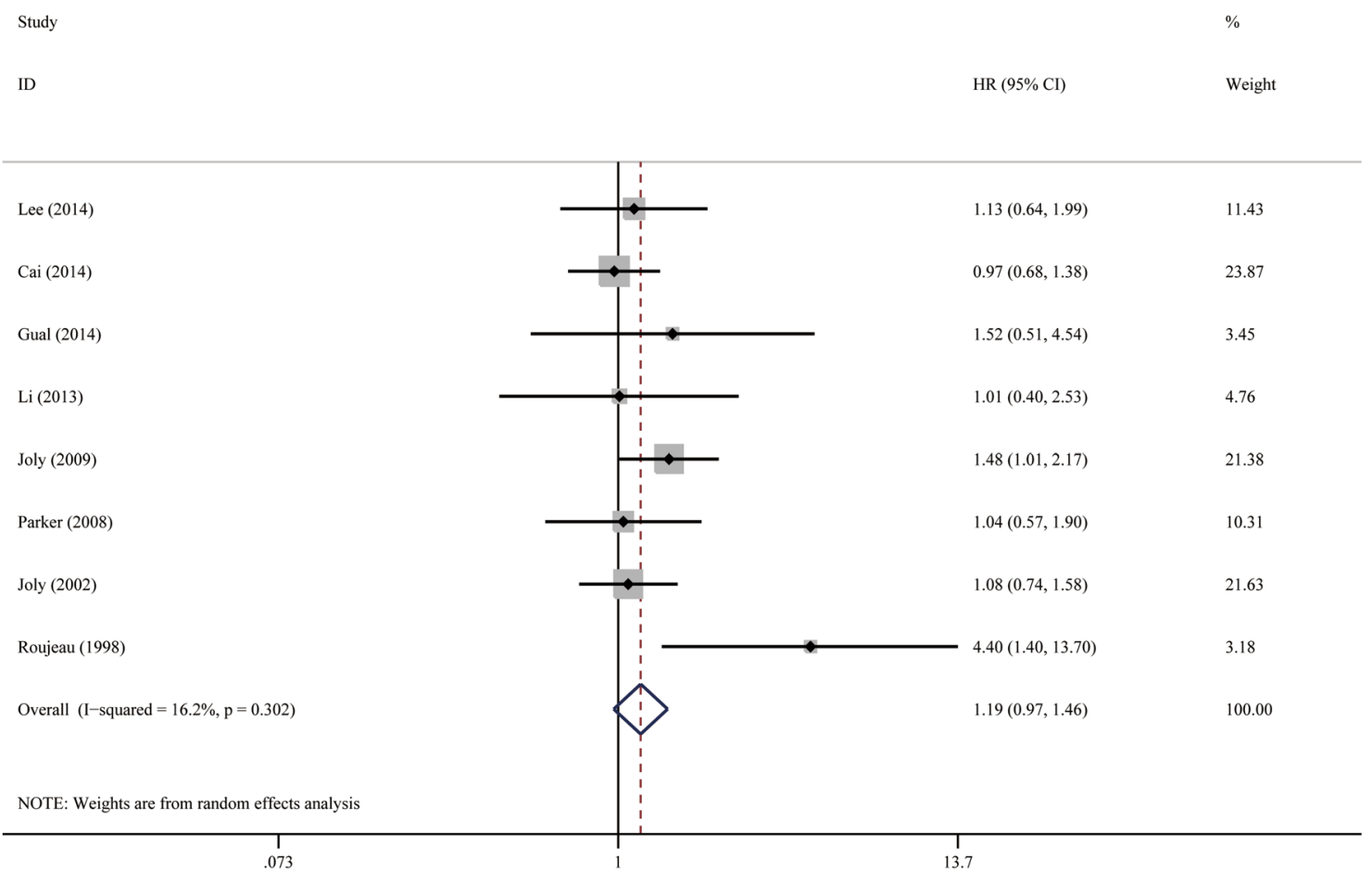

Fig. 4 Forest plots: association between bullous pemphigoid mortality and extensive disease

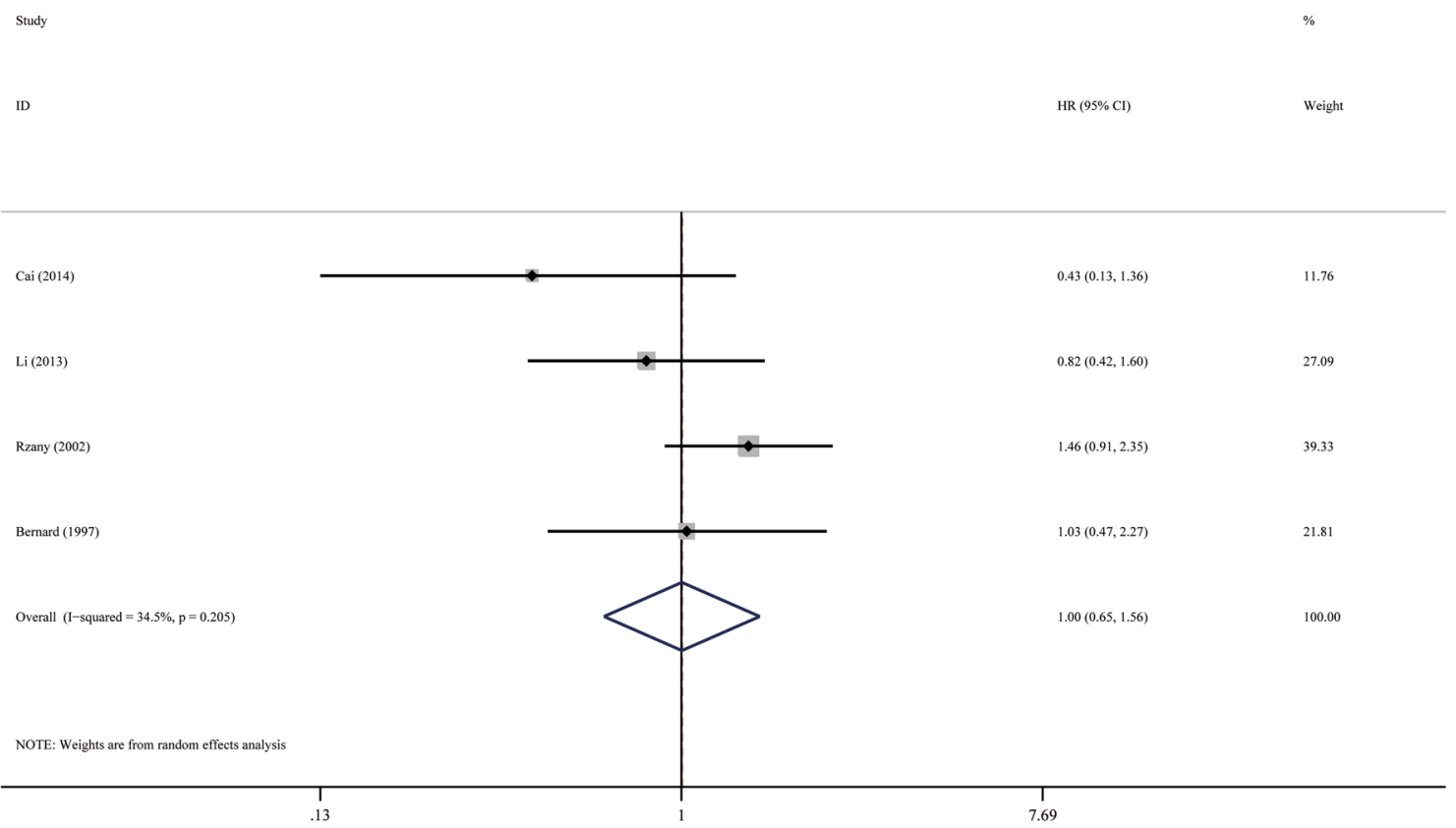

Fig. 5 Forest plots: association between bullous pemphigoid mortality and mucosal lesion

two outlier investigations by Roujeau et al. [29] and Cortés et al. [5] were small studies with less than 100 participants. Nevertheless, the above finding indicates that younger age at the diagnosis of BP does not necessarily denote a lower risk of mortality, and physicians should be more cautious about patients' general health condition and comorbidity profile.

As reported previously, more extensive form of BP did not significantly increase the risk on mortality. Although the extent and distribution of disease are a variable 
Study

ID
HR $(95 \%$ Cl)

$3.03(0.75,12.20)$

$1.78(0.54,5.85)$

$0.92(0.34,2.52)$

$2.23(0.50,9.98) \quad 6.79$

$1.43(0.69,3.01)$

$2.44(1.22,4.91)$

$1.77(1.20,2.61)-100.00$

Overall $(\mathrm{I}-\mathrm{squared}=0.0 \%, \mathrm{p}=0.632)$

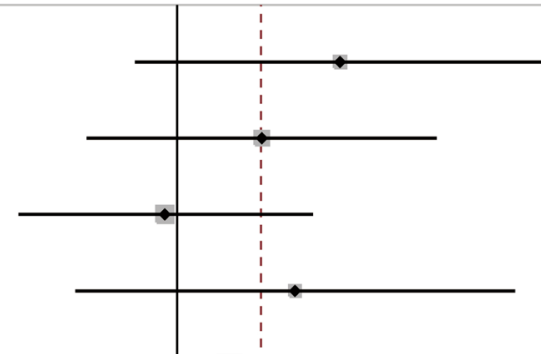

Joly (2005)

Bernard (1997)

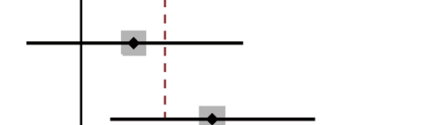

NOTE: Weights are from random effects analysis .082

Study

ID

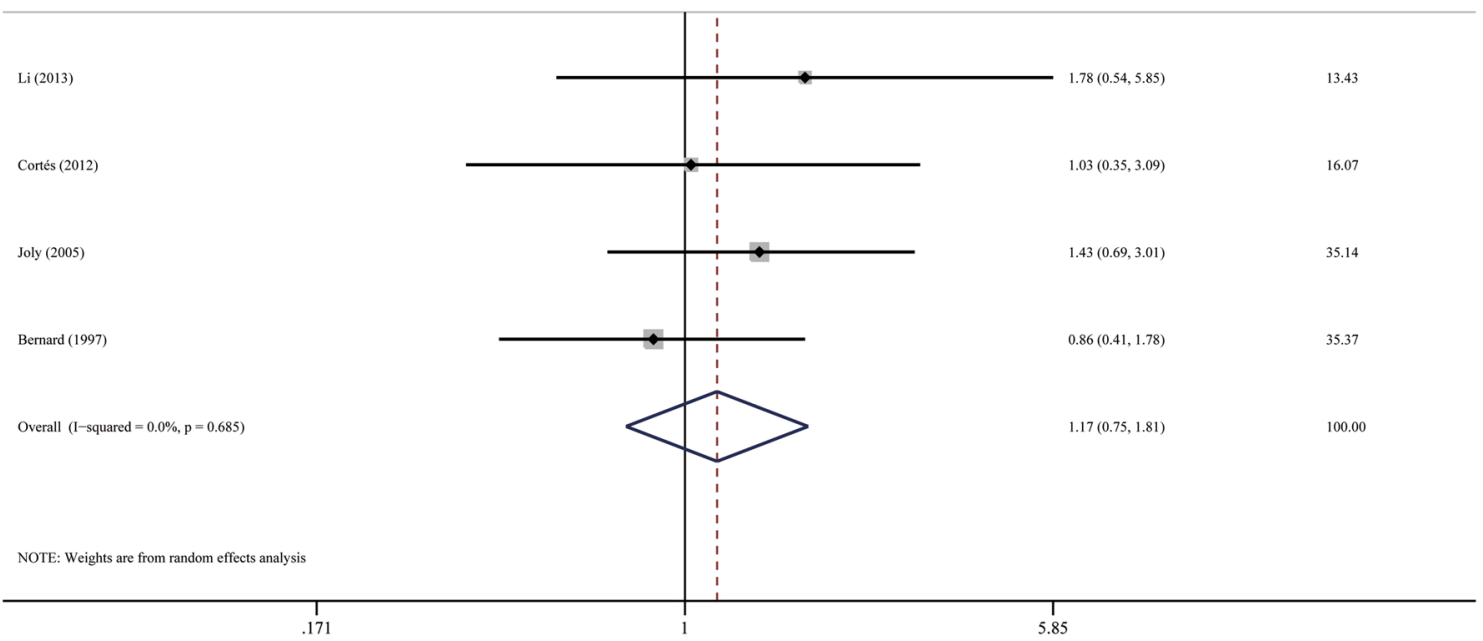

HR $(95 \% \mathrm{Cl}) \quad$ Weight

Fig. 6 Forest plots: association between bullous pemphigoid mortality and circulating BP autoantibody

Fig. 7 Forest plots: association between bullous pemphigoid mortality and positive IIF

susceptible to high variability between centers and may introduce information bias, especially in retrospective studies, heterogeneity between the included studies was low. The presence of mucosal lesion was also not predictive of poor survival outcome in our analysis. Of the other factors related to BP activity that were not included in this metaanalysis due to insufficient data for statistical pooling, such as pruritus [15, 29] and eosinophilia [1, 15, 22, 29], no mortality difference was found by previous investigations. These findings suggest that inherent factors such as age and 
Study

ID

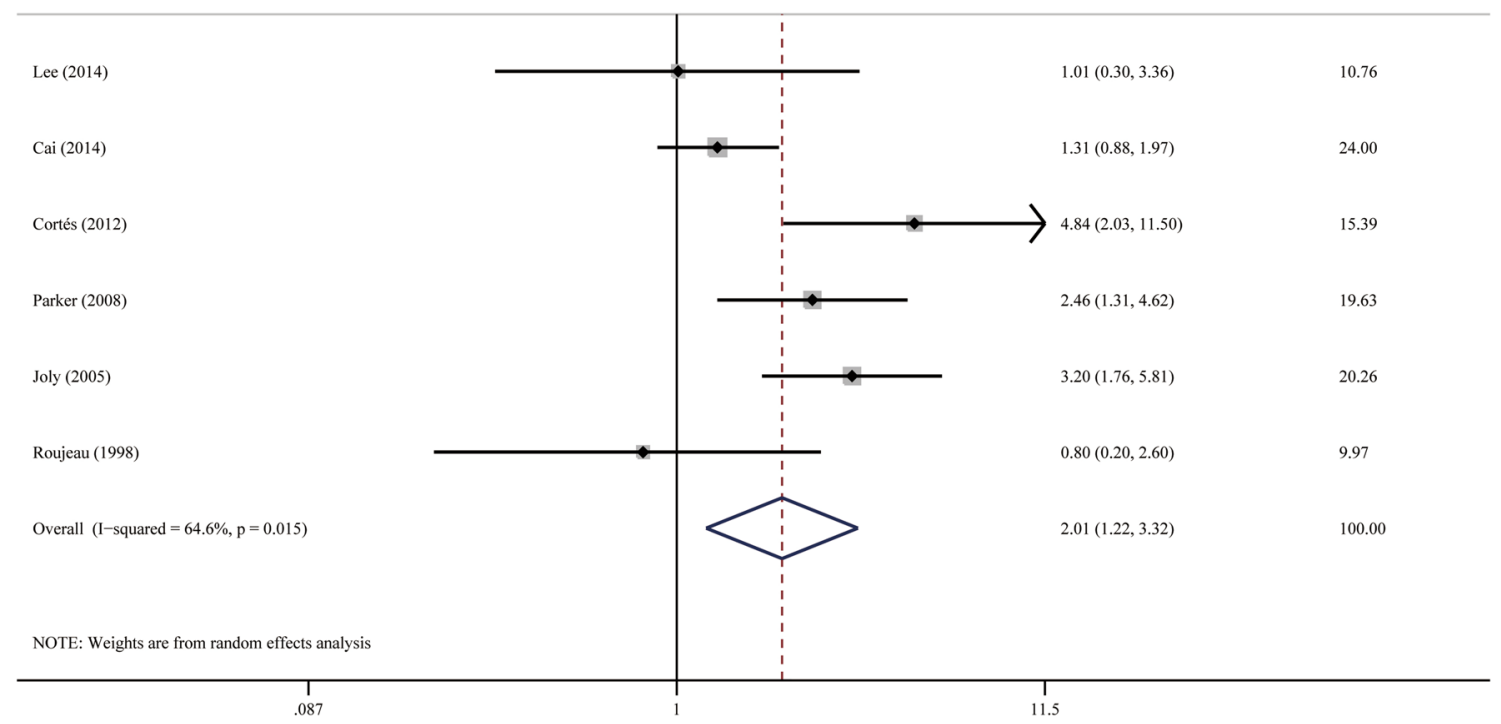

HR $(95 \%$ Cl) $\quad$ Weight

Fig. 8 Forest plots: association between bullous pemphigoid mortality and dementia

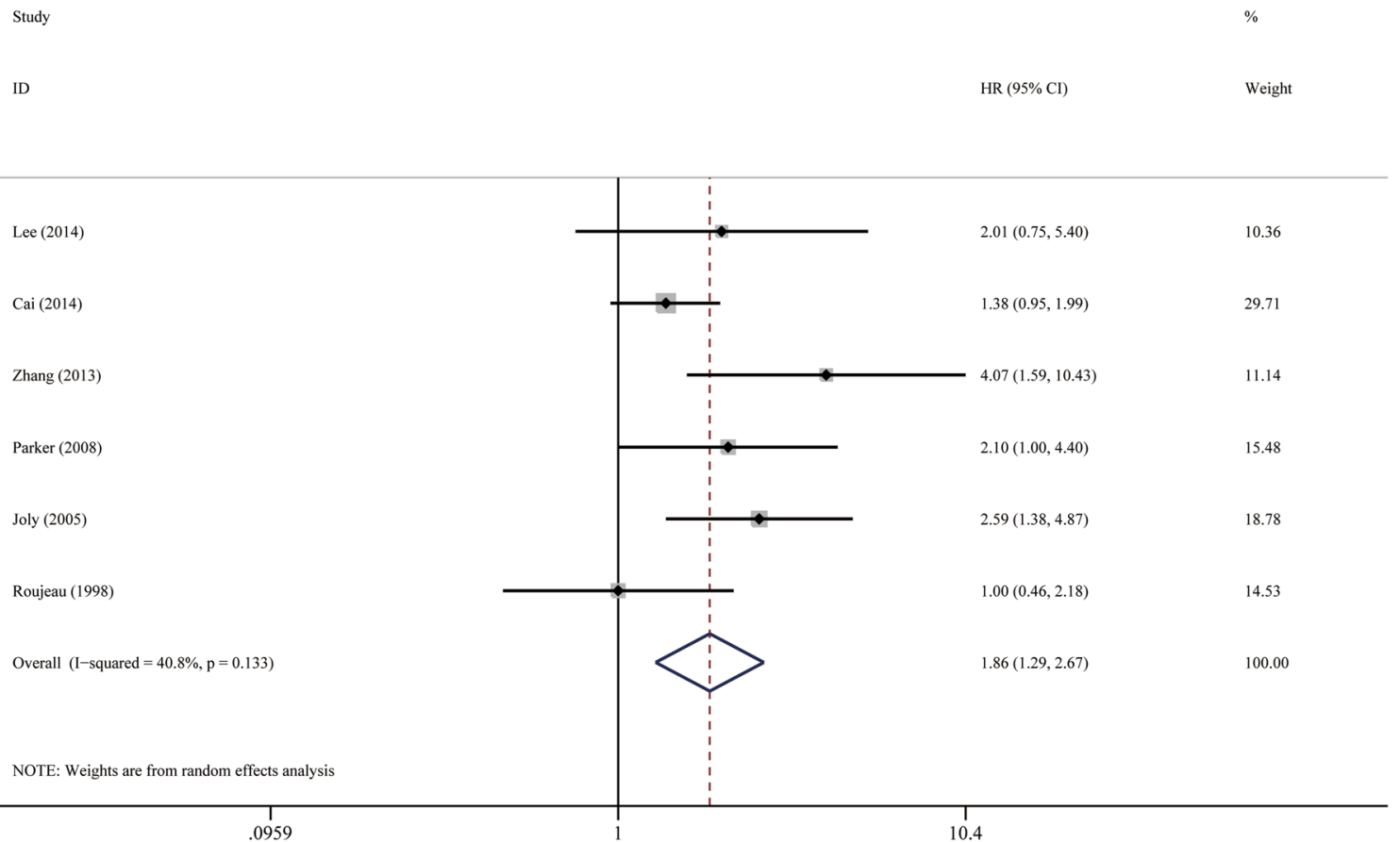

Fig. 9 Forest plots: association between bullous pemphigoid mortality and stroke

gender, as well as disease severity at the time of diagnosis, may not influence mortality as much as general condition and concomitant morbidities.

Our analysis corroborated previous finding that IIF is not a sensitive indicator of BP clinical activity [18], 

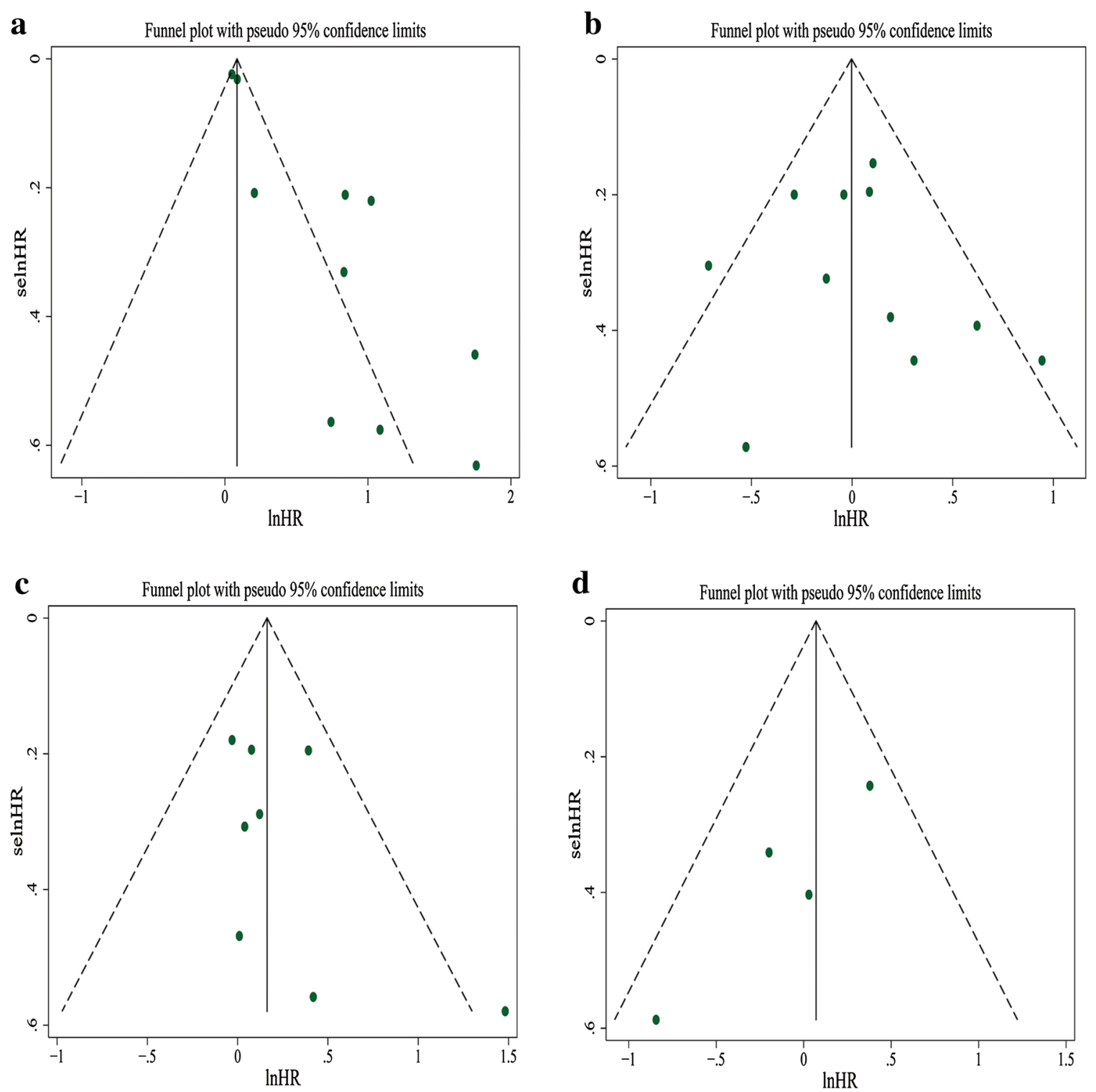

Fig. 10 Funnel plots of studies evaluating the relationship between bullous pemphigoid mortality and potential prognostic factors a old age, $\mathbf{b}$ gender (male vs female), $\mathbf{c}$ extensive disease, $\mathbf{d}$ mucosal lesion, $\mathbf{e}$ circulating autoantibody, $\mathbf{f}$ positive IIF, $\mathbf{g}$ dementia, $\mathbf{h}$ stroke

pooling for this. To date, IIF plays a key role in the detection of circulating antibodies and is often used as the routine test. However, the circulating autoantibodies can also be detected by more accurate serological assays, including immunoblotting and ELISA [18]. Of note, ELISA has been demonstrated as a useful tool to detect circulating antibodies in BP with its high sensitivity and specificity, particularly to recombinant BP180 antigen [18]. Moreover, it has the ability to reflect the severity of bullous pemphigoid, presumably because autoantibody against BP180 is believed to be pathogenic in BP [10]. It is noteworthy that this represents an intrinsic risk factor that correlates with BP mortality.
Severe concomitant morbidities at the time of diagnosis of bullous pemphigoid, such as neurological disorders, cardiac insufficiency, diabetes, and malignancies, increase the risk of mortality [3, 12, 21]. Interestingly, there is a strong link between BP and neurological diseases. In this study, we evaluated the impact of two most common neurological diseases in BP patients, dementia, and stroke, on the survival of BP patients. Our pooled data indicated a roughly two-fold higher mortality risk for BP patients with dementia or stroke. The exact mechanism underlying the link between neurological diseases and BP has not yet been fully elucidated, but a putative autoimmune reaction against $\mathrm{BP}$ antigens in the brain, 

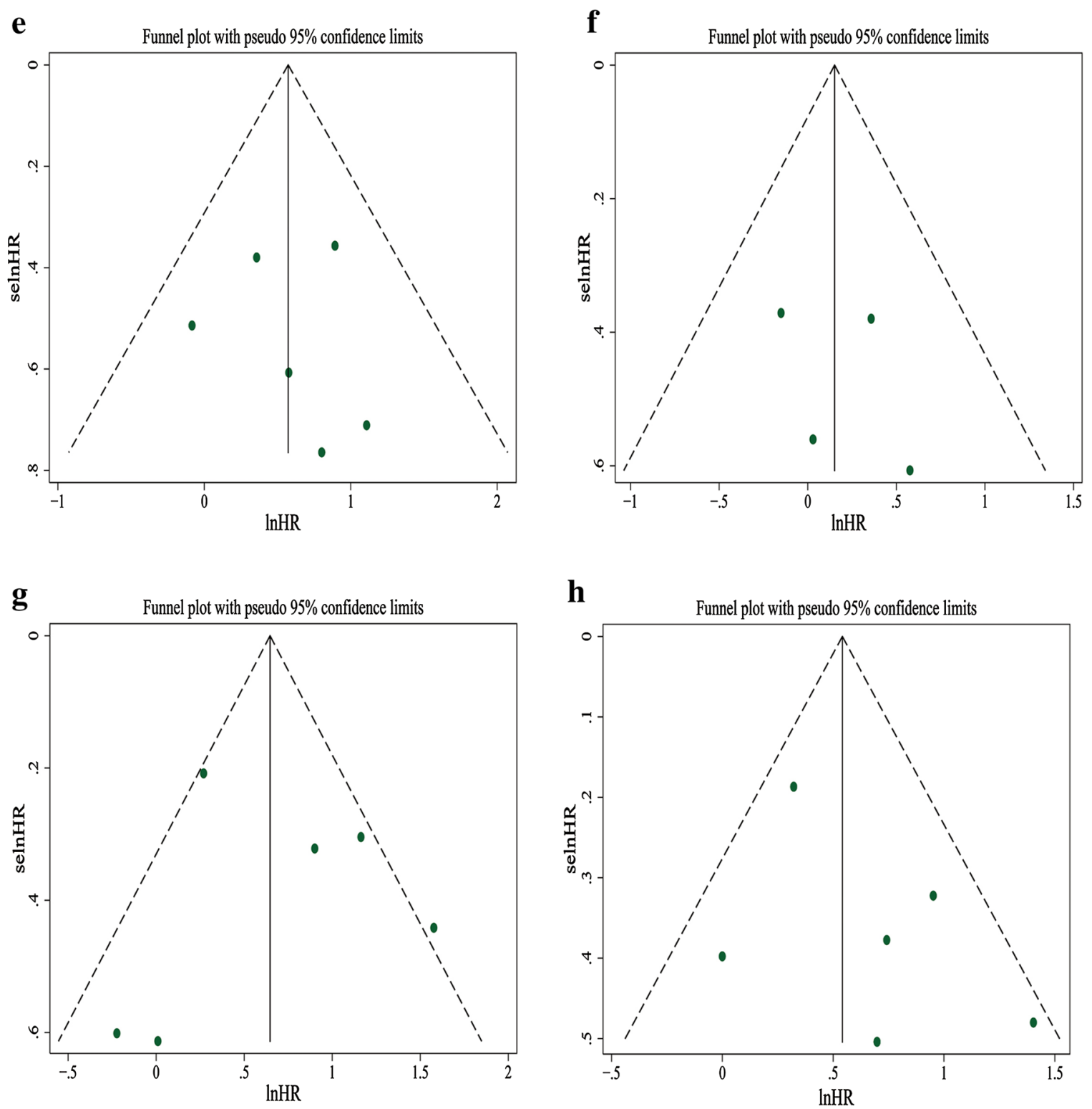

Fig. 10 (continued)

Table 3 Summary of pooled results of potential prognostic factors

\begin{tabular}{lllll}
\hline Prognostic factor & $\begin{array}{l}\text { Number of } \\
\text { studies }\end{array}$ & Participants & Hazard ratio (95\% CI) & Statistical difference \\
\hline Old age & 10 & 1922 & $1.63(1.34-1.97)$ & $\mathrm{S}(P<0.001)$ \\
Gender (male vs female) & 11 & 2016 & $1.01(0.81-1.25)$ & $\mathrm{NS}(P=0.944)$ \\
Extensive disease & 8 & 1861 & $1.19(0.97-1.46)$ & $\mathrm{NS}(P=0.102)$ \\
Mucosal lesion & 4 & 962 & $1.00(0.65-1.56)$ & $\mathrm{NS}(P=0.992)$ \\
Circulating autoantibody & 6 & 938 & $1.77(1.20-2.62)$ & $\mathrm{S}(P=0.004)$ \\
$\quad$ by any detection method & & & & \\
Positive IIF & 4 & 464 & $1.17(0.75-1.81)$ & $\mathrm{NS}(P=0.488)$ \\
Dementia & 6 & 1197 & $2.01(1.22-3.33)$ & $\mathrm{S}(P=0.006)$ \\
Stroke & 6 & 1231 & $1.86(1.29-2.67)$ & $\mathrm{S}(P<0.001)$ \\
\hline
\end{tabular}

$S$ significant, $N S$ non-significant, $I I F$ indirect immunofluorescence 
via a compromised blood-brain barrier, that cross react with the skin have been speculated as a possible cause [20]. Neurological diseases may be responsible for the poor prognosis of some patients with BP because of their altered general condition. However, patients with neurodegenerative disorders are known to have increased blood-brain barrier permeability that may facilitate the generation and crossing of BP autoantibodies into the periphery, worsening skin damage, and clinical outcome [8]. A recent study revealed that in a group of patients with Alzheimer's disease but no bullous pemphigoid, their sera contained much higher level of BP180 autoantibody than neurologically healthy controls. Furthermore, increased levels of the autoantibody were associated with more severe dementia [19]. Thus, it remains unclear whether neurological disease itself could increase the mortality risk, or the increased risk is conferred by functional impairment and decreased autonomy resulting from neurological disease. Future prospective studies should assess patient's functional status at initial presentation and employ a multivariate analysis to answer this question.

Meta-analysis of some other prognostic factors was not conducted, because the data were too sparse or heterogeneous to combine analytically. Poor general condition, low karnofsky scale, or being bedridden has been repeatedly reported to be associated with unfavorable outcome $[3,15,32]$, with the exception of one study conducted by Lee et al. [21], which did not find a significant association between karnofsky scale and BP mortality after multivariate analysis. Low serum albumin level and high erythrocyte sedimentation rate (ESR) were found to be associated with elevated mortality risk in BP $[22,30]$. High ESR may be a surrogate marker for more severe inflammatory status and more severe disease. Serum albumin level is related to patient's nutritional condition, or underlying diseases, or extensive cutaneous erosions. However, these two factors are easily influenced by various factors, such as other acute and chronic illnesses, so they do not represent intrinsic biological variables of bullous pemphigoid.

Steroid is the first line therapy in both localized and generalized BP [17]. High dosage of systemic corticosteroid or immunosuppressive agents correlates with higher mortality and increased side effects especially in patients at risk, as early deaths in BP patients are mainly of infectious or cardiovascular origin [16]. However, we were not in an optimal situation to validly investigate this issue, because (1) treatment modalities had not been predetermined and controlled in most studies in our analysis; (2) the average dosage of steroid used in different studies varied greatly; (3) dosage and route of administration were largely determined by patients' disease status; and (4) patients were often administered a combination of systemic steroid, topical steroid, and adjunctive immunomodulators.

Potential limitations need to be considered when interpreting the results of our meta-analysis. First, since most included studies were retrospective and conducted in referral centers, a potential unidentified confounding, information, and selection bias may exist, causing us to interpret our results with caution. Second, the follow-up time varied from 1 to 5 years among studies, which may influence the comparability of the results between component studies. Third, pooled results for some factors, such as mucosal involvement and positive IIF, were based on a limited number of studies. Therefore, we cannot rule out the possibility that insufficient statistical power is present. Finally, we could not evaluate all of the predictive factors for BP mortality in this meta-analysis.

\section{Conclusion}

Taken together, our results indicated that BP patients with older age, circulating BP autoantibodies, dementia, and stroke are at greater risk of mortality. Given that this conclusion was made using pooled data derived from multiple studies, such information has value for the purposes of patient education and prognosticating risk during the treatment process. Prognostic studies based on prospective design have the advantage of ruling out confounding effect, such as treatment regimen. More rigorously designed prospective studies with long-term follow-up duration are still required for future supplements and updates.

Acknowledgements This study was supported by the National Natural Science Foundation of China (81371731).

\section{Compliance with ethical standards}

Conflict of interest The authors declare that they have no conflict of interest.

Open Access This article is distributed under the terms of the Creative Commons Attribution 4.0 International License (http:// creativecommons.org/licenses/by/4.0/), which permits unrestricted use, distribution, and reproduction in any medium, provided you give appropriate credit to the original author(s) and the source, provide a link to the Creative Commons license, and indicate if changes were made.

\section{References}

1. Bernard P, Bedane C, Bonnetblanc JM (1997) Anti-BP180 autoantibodies as a marker of poor prognosis in bullous pemphigoid: a cohort analysis of 94 elderly patients. $\mathrm{Br} \mathrm{J}$ Dermatol 136:694-698 
2. Brick KE, Weaver CH, Lohse CM, Pittelkow MR, Lehman JS, Camilleri MJ, Al-Hashimi M, Wieland CN (2014) Incidence of bullous pemphigoid and mortality of patients with bullous pemphigoid in Olmsted County, Minnesota, 1960 through 2009. J Am Acad Dermatol 71:92-99

3. Cai SC, Allen JC, Lim YL, Chua SH, Tan SH, Tang MB (2014) Mortality of bullous pemphigoid in Singapore: risk factors and causes of death in 359 patients seen at the National Skin Centre. Br J Dermatol 170:1319-1326

4. Colbert RL, Allen DM, Eastwood D, Fairley JA (2004) Mortality rate of bullous pemphigoid in a US medical center. J Invest Dermatol 122:1091-1095

5. Cortés B, Khelifa E, Clivaz L, Cazzaniga S, Saurat JH, Naldi L, Borradori L (2012) Mortality rate in bullous pemphigoid: a retrospective monocentric cohort study. Dermatology 225:320-325

6. Cortés B, Marazza G, Naldi L, Combescure C, Borradori L (2011) Mortality of bullous pemphigoid in Switzerland: a prospective study. Br J Dermatol 165:368-374

7. DerSimonian R, Laird N (1986) Meta-analysis in clinical trials. Control Clin Trials 7:177-188

8. Desai BS, Monahan AJ, Carvey PM, Hendey B (2007) Bloodbrain barrier pathology in Alzheimer's and Parkinson's disease: implications for drug therapy. Cell Transpl 16:285-299

9. Egger M, Davey SG, Schneider M, Minder C (1997) Bias in meta-analysis detected by a simple, graphical test. BMJ 315:629-634

10. Fichel F, Barbe C, Joly P, Bedane C, Vabres P, Truchetet F, Aubin F, Michel C, Jegou J, Grange F, Antonicelli F, Bernard P (2014) Clinical and immunologic factors associated with bullous pemphigoid relapse during the first year of treatment: a multicenter, prospective study. JAMA Dermatol 150:25-33

11. Försti AK, Jokelainen J, Timonen M, Tasanen K (2016) Risk of death in bullous pemphigoid: a retrospective database study in Finland. Acta Derm Venereol 96:758-761

12. Gual A, Mascaro JJ, Rojas-Farreras S, Guilabert A, Julia M, Iranzo P (2014) Mortality of bullous pemphigoid in the first year after diagnosis: a retrospective study in a Spanish medical centre. J Eur Acad Dermatol Venereol 28:500-506

13. Higgins JP, Thompson SG, Deeks JJ, Altman DG (2003) Measuring inconsistency in meta-analyses. BMJ 327:557-560

14. Joly P, Baricault S, Sparsa A, Bernard P, Bedane C, DuvertLehembre S, Courville P, Bravard P, Remond B, Doffoel-Hantz V, Benichou J (2012) Incidence and mortality of bullous pemphigoid in France. J Invest Dermatol 132:1998-2004

15. Joly P, Benichou J, Lok C et al (2005) Prediction of survival for patients with bullous pemphigoid: a prospective study. Arch Dermatol 141:691-698

16. Joly P, Roujeau JC, Benichou J, Picard C, Dreno B, Delaporte E, Vaillant L, D'Incan M, Plantin P, Bedane C, Young P, Bernard $\mathrm{P}$ (2002) A comparison of oral and topical corticosteroids in patients with bullous pemphigoid. N Engl J Med 346:321-327

17. Joly P, Roujeau JC, Benichou J et al (2009) A comparison of two regimens of topical corticosteroids in the treatment of patients with bullous pemphigoid: a multicenter randomized study. J Invest Dermatol 129:1681-1687
18. Kershenovich R, Hodak E, Mimouni D (2014) Diagnosis and classification of pemphigus and bullous pemphigoid. Autoimmun Rev 13:477-481

19. Kokkonen N, Herukka SK, Huilaja L, Kokki M, Koivisto AM, Hartikainen P, Remes AM, Tasanen K (2016) Increased levels of the bullous pemphigoid BP180 autoantibody are associated with more severe dementia in Alzheimer's disease. J Invest Dermatol. doi:10.1016/j.jid.2016.09.010

20. Lai YC, Yew YW, Lambert WC (2016) Bullous pemphigoid and its association with neurological diseases: a systematic review and meta-analysis. J Eur Acad Dermatol Venereol 30:2007-2015

21. Lee JH, Kim SC (2014) Mortality of patients with bullous pemphigoid in Korea. J Am Acad Dermatol 71:676-683

22. Li J, Zuo YG, Zheng HY (2013) Mortality of bullous pemphigoid in China. JAMA Dermatol 149:106-108

23. Marazza G, Pham HC, Scharer L, Pedrazzetti PP, Hunziker T, Trueb RM, Hohl D, Itin P, Lautenschlager S, Naldi L, Borradori L (2009) Incidence of bullous pemphigoid and pemphigus in Switzerland: a 2-year prospective study. Br J Dermatol 161:861-868

24. McPheeters ML, Kripalani S, Peterson NB, Idowu RT, Jerome RN, Potter SA, Andrews JC (2012) Closing the quality gap: revisiting the state of the science (vol. 3: quality improvement interventions to address health disparities). Evid Rep Technol Assess (Full Rep) 208.3:1-475

25. Moher D, Liberati A, Tetzlaff J, Altman DG (2010) Preferred reporting items for systematic reviews and meta-analyses: the PRISMA statement. Int J Surg 8:336-341

26. Mor V, Laliberte L, Morris JN, Wiemann M (1984) The Karnofsky Performance Status Scale. An examination of its reliability and validity in a research setting. Cancer 53:2002-2007

27. Parker SR, Dyson S, Brisman S, Pennie M, Swerlick RA, Khan R, Manos S, Korman BD, Xia Z, Korman NJ (2008) Mortality of bullous pemphigoid: an evaluation of 223 patients and comparison with the mortality in the general population in the United States. J Am Acad Dermatol 59:582-588

28. Ren Z, Hsu DY, Brieva J, Silverberg NB, Langan SM, Silverberg JI (2016) Hospitalization, inpatient burden and comorbidities associated with bullous pemphigoid in the U.S.A. Br J Dermatol. doi:10.1111/bjd.14821

29. Roujeau JC, Lok C, Bastuji-Garin S, Mhalla S, Enginger V, Bernard P (1998) High risk of death in elderly patients with extensive bullous pemphigoid. Arch Dermatol 134:465-469

30. Rzany B, Partscht K, Jung M et al (2002) Risk factors for lethal outcome in patients with bullous pemphigoid: low serum albumin level, high dosage of glucocorticosteroids, and old age. Arch Dermatol 138:903-908

31. Tierney JF, Stewart LA, Ghersi D, Burdett S, Sydes MR (2007) Practical methods for incorporating summary time-to-event data into meta-analysis. Trials 8:16

32. Zhang LM, Wu J, Xiao T, Jin GY, Li JH, Geng L, He CD, Gao $\mathrm{XH}$, Chen HD (2013) Treatment and mortality rate of bullous pemphigoid in China: a hospital-based study. Eur J Dermatol 23:94-98 